\title{
Micrandra scleroxylon W. Rodr., nova Euforbiácea da Amazônia Brasileira
}

\author{
WILLIAM A. RODRIgUes (*) \\ Instituto Nacional de Pesquisas \\ da Amazônia
}

\begin{abstract}
SINOPSE
Uma árvore relativamente abundante das matas de terra firme das cercanias de Manaus, Amazonas, é descrita como nova para a Amazônia sob o binômio de Micrandra scleroxylon W. Rodr. (família Euphorbiaceae). Juntam-se à diagnose latina alguns dados dendrológicos e ecológicos do nôvo taxon, a'ém da descrição das características gerais de sua madei$\mathrm{ra}$, em especial do ponto de vista macroscópico.
\end{abstract}

\section{INTRODUÇÃO}

Desde a implantação do INPA na região amazônica temos realizado intensivo levantamento florístico de Manaus e arredores, admitindo que sua área deva ser considerada prioritária para êste tipo de pesquisa. por supor que o conhecimento imediato de sua flórula é de suma importância não só do ponto de vista puramente científico como aplicado, antes que o resto de mata primária ainda existente em suas cercanias desapareça totalmente. A destruiçãc de sua mata é um fato aceito sem contestação e aí já se vem sentindo muito mais intensamente que em qualquer outro lugar, devido às peculiaridades especiais que oferece Manaus como um dos mais importantes polos naturais de desenvolvimento da Amazônia Ocidental.

Em razão de tais estudos, vários "taxa" novos ou pouco conhecidos têm sido encontrados e revelados por nós e outros colaboradores. A nova espécie que aqui apresentamos é mais um resultado dessas pesquisas.

\section{DESCRIÇÃo LATINA}

Micrandra scleroxylon $\mathrm{W}$. Rodrigues, $\mathrm{n} . \mathrm{sp}$.

(fig. 1 e 2)

Arbor monoica ad $25 \mathrm{~m}$ alta et $55 \mathrm{~cm}$ diametro vulgo radicibus tabularibus distituta truncus erectus, columnaris; cortex tenuis, 2-5 $\mathrm{mm}$ crassus, extus fusco-nigrescens, in statu vivo intus carneo, siccitate brunneo, succo exiguo lactoso-albo praeditus; duramen fuscobrunneum, durissimum; ramuli terminales glabri, nigrescentes, in vivo virescentes, subteretes, $0,3-0,5 \mathrm{~cm}$ diametro, longitudinaliter striati. Folia chartacea vel subcoriacea, oblonga, lanceolata vel oblongo-lanceolata, ad apicem abrupte acuminata vel cuspidata, distincte mu cronata, mucro nigrescens $1,4-2,5 \mathrm{~mm}$ longus, 0,6-1,0 $\mathrm{mm}$ crassus, ad basin obtusa vel rotun. data. Stipula caducissima triangulari-lanceola. ta, subulata, pubescens ca. $1,5 \mathrm{~cm}$ longa. Petioli fusco-nigrescentes, $1,2-2,0 \mathrm{~cm}$, longi 1,5-2,5 mm crassi, apicem versus leviter tumidi, supra caniculati. Lamina foliorum $11-30 \mathrm{~cm}$ longa, 3-9 cm lata, margine integra, utrinque glaberrima, discolor, supra atrovirens, nitidula, subtus pallidior (siccitate subtus fusca, supra paullo pallidior vel pallide virescens) juxta basin costae superiores uniglandulosa vel indistincte biglandulosa (glandula verruciformis (rassa, nigra); costae centrales utrinque prominentes; venae secundariae $11-18$, jugae, supra leviter elevatae, subtus prominentes. arcuato-adscendentes, prope marginem tenuiter anastomosantes; venulae tenues reticulatae, subtus prominulae. Inflorescentiae terminales et axillares paniculatae, folia ca. duplo superantes, pendulae, distincte longiramosae. usque ad $45 \mathrm{~cm}$ longae; rachis florifera in vivo virescens, dense stellato-pilosa. Bracteae 2.6 $\mathrm{mm}$ longae, $1,5 \mathrm{~mm}$ latae, triangulari-lanceolatae, pubescentes ad basin glandulosae, glandulis magnis in vivo virescentibus. Bracteolae bracteis similes eis multo minores. Pedicelii microscopice pubescentes usque ad $5 \mathrm{~mm}$ longi

(*) - Bolsista do Conselho Nacional de Pesquisas. 


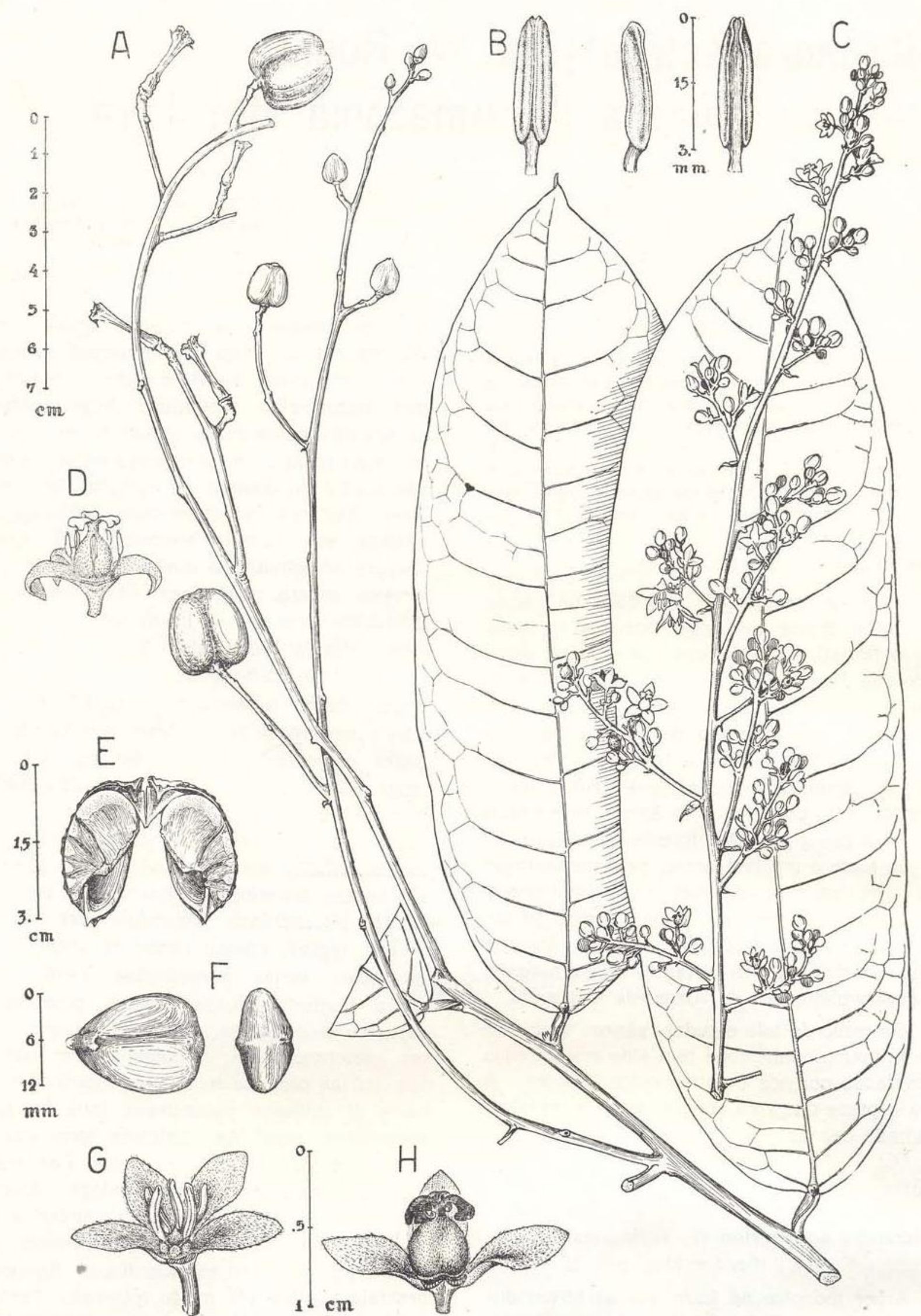

Fig. 1 - Micrandra scleroxylon W. Rodr., n. sp. A - Hábito de um raminho fértil. $B$ - Antera vista de frente, perfil e costa. $C$ - Inflorescência. $D$ - Flor perfeita. $E-$ Duas valvas da cápsula. $F-$ Semente vista de frente e em seção transversal. $G-$ Flor masculina. $H-$ Flor feminina. (Desenhos de J. Dellome) 
Alabastra evoluta oblonga, ovoidea vel globosoelongata, microscopice cinereo-pubescentia, ca. 2-5 mm longa, 2-3 $\mathrm{mm}$ diametro. Flores flavescentes, saepissime unisexuales, subinde hermaphod!ti. Calyces florum masculorum 5 laciniis lanceolato-ovatis vel oblongo-ovatis. subaequalibus, imbricatis, crassi ad apicem obtusis vel acutis, extus puberulis intus dense pubescentibus $3-5 \mathrm{~mm}$ longis, $1,5-3 \mathrm{~mm}$ latis; florum feminorum 5 laciniis cito deciduis, lanceolato-ovatis vel oblongo-ovatis, puberulis, subaequalibus, $5-7 \mathrm{~mm}$ longis, $2,0-4 \mathrm{~mm}$ latis. Antherae 5-7, saepius 5 , flavae, alongatae, $2,0-3 \mathrm{~mm}$ longae, $0,4-0,5 \mathrm{~mm}$ latae, rimosae. Filamenta 0,5-1,0 $\mathrm{mm}$ longa subdisco depresso 5-lobato inserta, libera; dicus florum masculorum glanduloso-lobatus, in vivo roseolus, sicco dense fulvo-sericeus. Ovarium fulvo-sericeum, ovoideum, ad apicem abrupte acuminatum 3-4 $\mathrm{mm}$ longum, 2-3,5 $\mathrm{mm}$ diametro, disco hypogyno annulari tenuissimo; stilus subsessilis in vivo flavescens, sicco nigeri, tripartius, applanatus, cum divisionibus bifidis, crassis, usque ad $2 \mathrm{~mm}$ longis, 1,5-2 $\mathrm{mm}$ latis supra glabris, subtus pubescentibus, basi distincte connatis. Fructus subgloboso-trigastricus, in vivo (ut siccitate) usque ad $2,4 \mathrm{~cm}$ longus, $2,5 \mathrm{~cm}$ latus, ad apicem rotundato-obtusus leviter apiculatus, ad basin plus $\mathrm{mi}$ nus truncatus; epicarpio viridi sed maturitate flavescente, sparse microscopiceque stellatopiloso, siccitate rugoso; mesocarpio carnoso tenuissimo, siccitate superficie interna dense alveolato; endocarpio lignoso usque ad $2,5 \mathrm{~mm}$ crasso, valvis regularibus $2,4 \mathrm{~cm}$ longis, $1,2 \mathrm{~cm}$ latis. Pedicello fructifero terete ca. $1,0 \mathrm{~cm}$ longo, $0,3 \mathrm{~cm}$ diametro. Semina carunculata (in vivo caruncula usque ad $0,4 \mathrm{~cm}$ lata, in sicco $0,3 \mathrm{~cm}$ ) ovata, testa badio-brunnea, nitida, $1,3-1,7 \mathrm{~cm}$ longa, $1,0-1,2 \mathrm{~cm}$ lata, leviter compressa, $0,6-0,8 \mathrm{~cm}$ crassa in ambitu transversali inaequaliter rhomboidea, carina dorsali conspicua. Plantula phanerocotylar, lacto-succosa, radice primaria recta usque ad ca. $17 \mathrm{~cm}$ longa radicellis paulo multiplicibus verticillatis; hypacotylo glabro, modice angulari, siccitate longitudinale striati, laevi, viridi, ca. $2 \mathrm{~mm}$ diametro. Cotyledones 2 opposites subsessiles, la. minis obovatis subchartaceis apice rotundatis basi biglandulosis, siccitate, ca $3,5-5,5 \mathrm{~cm}$ longis, $2,5-4,5 \mathrm{~cm}$ latis, opacis, discoloribus, supra atroviridibus, infra pallidioribus. margine inte- gra, emarginatis, trinervis, nervis venisque utrinque prominulis, venis reticulatis juxta marginem anastomosantibus; petioli modice crassi fusco-nigrescentes usque ad $0,3 \mathrm{~cm}$ longi. Epicotylus laevis angulosus sulcatus modice reflexus, nodicis inter se $0,6-3,5 \mathrm{~cm}$ longis. Eophylae lamina anguste lanceolata alterna, discolor, margine integra, circiter $8-13 \mathrm{~cm}$ longa, ca. $1,5-3,5 \mathrm{~cm}$ lata, apice attenuato-cuspidata vel modice abrupte cuspidata, ad basin longe cuneata, eglandulosa vel vix conspicue biglandulosa, penninervia; costa primaria, nervis venisque utrinque prominulis, infra stramineis, nervis adscendentibus alternis inter se sub-marginem tenuiter anastomosantibus, venis dense reticulatis; petiolo tenui, caniculato, fulvo, glaberrimo

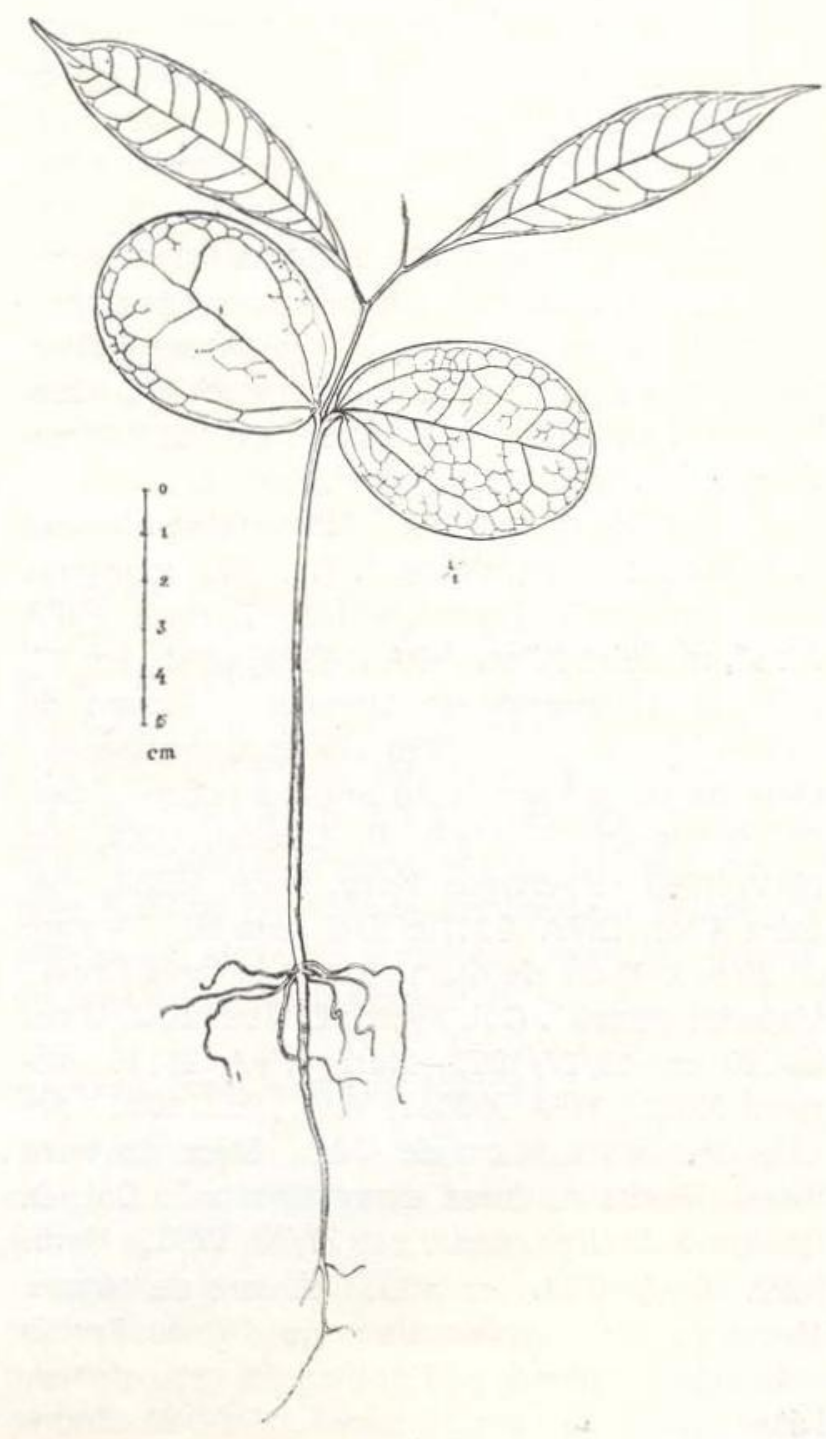

Fig. 2 - Hábito de uma plântula de Micrandra scleroxvlon W. Rodr. n. sp. (Des. Júlio Dellome Filho) 
6-12 $\mathrm{mm}$ longo paene $0,5 \mathrm{~mm}$ crasso; stipulis caducissimis, puberulis, triangularibus, subulatis paene $1 \mathrm{~mm}$ longis latisque.

Habitat in vicinia Manaus, Amazonas, ubi satis frequens in silva primaria non inundabili, ab incolis "acapuri" nuncapatur. W. A. Rodrigues, F. Mello, D. Coelho, 6961, typus (Holotypus INPA 15534, Isotypus NY, MG, ECON).

\section{MIATERIAL DE REFERÊNCIA}

BRASIL : Estado do Amazonas, Manaus, igarapé do Tabatinga. "Árvore de $10 \mathrm{~m} \mathrm{x} 8 \mathrm{~cm}$ da mata de terra firme, argilosa. Frutos amarelo-esverdeados. Madeira amarelada, dura, pesada, casca fina, pouco rugosa". Col. L. Coelho \& F. Mello, s/n. ${ }^{\circ}$ em 2/XII/1955, Herb. INPA 3005, madeira Xiloteca INPA X-388; Estrada Manaus-Itacoatiara, $\mathrm{Km}$ 43. "Árvore de $7 \mathrm{~m}$ da mata de terra firme, solo argiloso. Frutos amarelos". Col. W. Rodrigues \& D. Coelho 1381, em 26/XI/1959. Herb. INPA 7711; ibid. Km 55. "Árvore de $20 \mathrm{~m}$ da mata de terra firme; latex branco. Inflorescência amarela aromática. Frutos maduros e jovens. Madeira creme (alburno) de casca fina". Col. W. Rodrigues \& L. CoeIho, 1844 , em 14/X/1960. Herb. INPA 8216, madeira Xilot. INPA X-798; ibid., km 66. "Árvore de $15 \mathrm{~m} \times 25 \mathrm{~cm}$ da mata. Flôres amarelas em inflorescêricias pendentes". Col. W. Rodrigues \& A. Loureiro, 7065, em 1/VIII/1965. Herb. INPA 15727, madeira Xilot. INPA X-3228; obid., km 27, próximo do igarapé do Mariano. "Árvore de $12 \mathrm{~m} \times 25 \mathrm{~cm}$ diâm. com flôres esverdeadas. Mata de terra firme, solo argilo-arenoso". Col. W. Rodrigues, F. Melo, D. Coelho, 6961, em 29/VI/1965. Holotypus Herb. INPA 15534, madeira Xilot. INPA X-3170; ibid., km 90. "Árvore de $20 \mathrm{~m} \times 40 \mathrm{~cm}$ de diâm. Mata de terra firme. Material estéril". Col. Byron, L. Coelho, J. Lima, 68-120 em 19/IV/1968. Herb. INPA 21146, madeira Xilot. INPA X-3851; ibid., km 108. "Árvore de $12 \mathrm{~m} \times 15 \mathrm{~cm}$ de diâm. Mata de terra firme. Frutos maduros avermelhados". Col. D. Coelho \& J. Lima, s/n. ${ }^{\circ}$ em 27/XI/1968. Herb. INPA 25949; ibid., km 130. "Árvore de $10 \mathrm{~m} \times$ $18 \mathrm{~cm}$ de diâm., mata de terra firme. Frutos amarelo-esverdeados. Frutificaçã́o abundante. Látex muito escasso, branco". Col. W. Rodrigues 8639, em 12/XI/1969; ibid., km 26, igarapé do Mariano. "Árvore de $18 \mathrm{~m} \times 15 \mathrm{~cm}$ D.A.P. na margem alta do igarapé. Frtitos maduros" Col. J. Aluisio 304, em 3/XII/1969; ibid., km 68. "Árvore mediana bastante freqüente na mata de terra firme. Látex branco muito escasso. Tronco sem sapopema. Em fim de frutificação. Mudas abundantes em redor da árvore, de folhas primárias nítidas e verde-escuras na parte superior. Nome vulgar: Acapuri". Col. W. Rodrigues 8732, em 5/III/1970. Herb. INPA 27906; ibid., km 114, lado esquerdo. "Árvore de $15 \mathrm{~m}$ x $20 \mathrm{~cm}$ de diâm., bastante freqüente na mata de terra firme; ocasional na margem da estrada. Bracteolas amareladas. Flôres amareladas aromáticas em inflorescências pendentes. Floração abundante. Flôres unissexuais, às vezes hermafroditas. Pedúnculo floral verde-ciaro, Cálice carnoso com pêlos brancos na face ventral. Flôres masculinas com anteras e filêtes amarelos, êstes com pêlo branco até o conectivo; disco róseo, piloso; ovário rudimentar creme-esverdeado. Flôres femininas com ovário esverdeado; estigma verde, depois amarelo; disco delgado alaranjado soldado ao ovário. Fruto amarelo $\mathrm{ccm}$ pêlos estrelados diminutos e esparsos". Col. W. Rodrigues 8982, em 23/X/1970. Herb. INPA 28368.

\section{DADOS GERAIS}

Esta interessante espécie distingue-se de qualquer outra congênere, dentre outros caracteres, pela fôlha elíptica, uma única glândula foliar verrucıforme na base do limbo, brácteas glanduliferes, anteras alongadas e madeira castanho-escura, muito dura.

A glândula foliar única, que anormalmente aparece na base do limbo parece ser formada pela fusão de duas glândulas distiıtamente observáveis nos eófilos e malmentc em alguils metáfilos. Anteras alongadas, verificadas na nova espécie, são características de outros gêneros de Euforbiáceas como Pogonophora Miers, Jatropha L., Manihot Miller etc.,- porém até então estranhas para o gênero Micrandra Benth.

Flôres unissexuais é normal na espécie, porém não é raro encontrarmos flôres morfológicas e funcionalmente perfeitas numa mesma planta, curiosa estrutura também já assinalada por Schultes (1952) em Micrandra lopezii R. E. Schultes. 
O pólen é tricolporado às vêzes tetracolporado. A forma tricolporada não diverge do que observou Punt (1962) e G. Webster, citado por Schultes (1955) para outras espécies do gênero.

O seu habitat é a mata de terra firme de solo argiloso. Nunca a encontranios ein matas pantanosas nem pseudocaatingas ou carrascos de solo humo-silicoso, como acontece com a maioria de suas congêneres.

Não é geralmente de grande porte nem avantajado diâmetro de fuste. As maiores árvores năo passam do dossel da mata, isto é, cerca de $25 \mathrm{~m}$ de altura. Inventários florestais realizados pelo autor (1967) na estrada ManausItacoatiara indicaram que a maioria não ultrapassava a classe de diâmetro de fuste de $25-34 \mathrm{~cm}(83 \%)$, ficando o restante nas classes de $35-44 \mathrm{~cm}(12,5 \%)$ e $45-54 . \mathrm{cm}(4,5 \%)$. O volume médio de madeira comerciável por hectare foi de $0,525 \mathrm{~m}^{3}$, considerando apenas as árvores acima de $25 \mathrm{~cm}$ de diâmetro de fuste acıma do peito.

Raízes tabulares grandes não foram vistas na espécie. Em geral as raízes são axonomorfas.

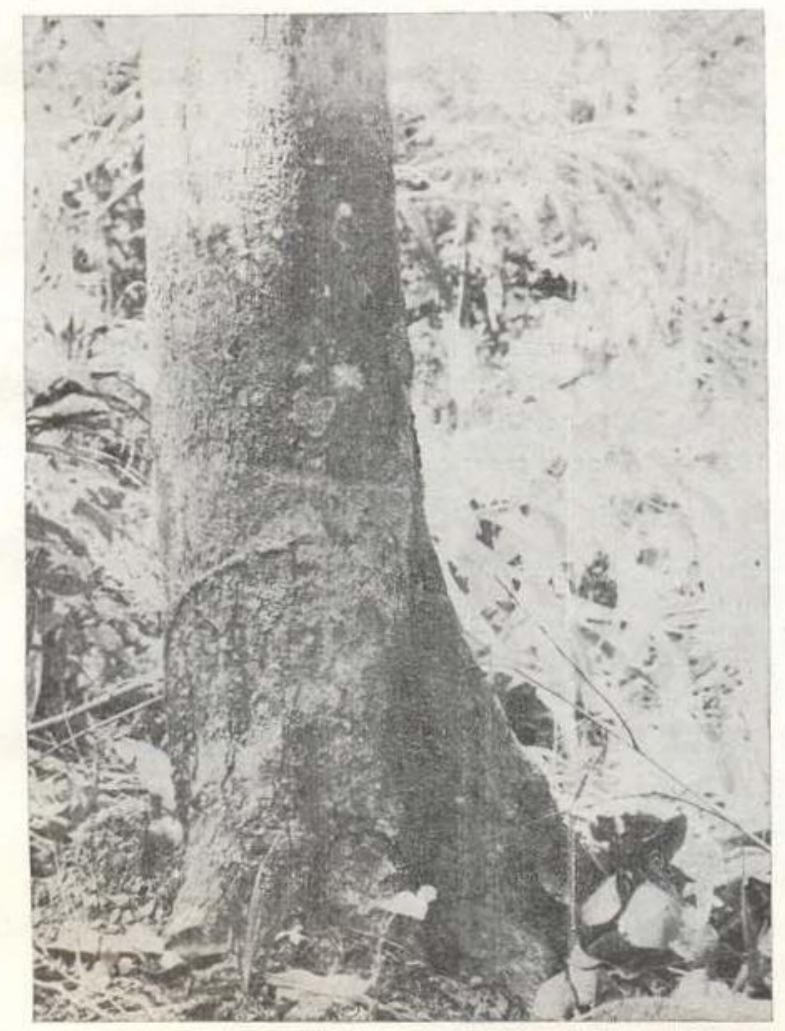

Fig. 3 - Base do tronco de Micrandra scleroxylon W. Rodr. n. sp'
Ecologicamente, Micrandra scleroxylon parece mostrar um grau elevado de tolerância à sombra da mata local, haja vista termos visto um grande número de indivíduos ocupando diferentes andare's da mata, alguns inclusive aí florescendo e frutificando reguiarmente. O seu poder de regeneração é um tanto elevado, sendo comum encontrarmos uma boa quantidade de mudinhas recém-germinadas em tôrno das árvores-matrizes.

Do ponto de visto morfológico, a plântula (fig. 2) não diverge em nada das outras espécies afins do gênero, em especial de Micrandra siphonioides Bth. e M. elata (Didrichs) M. Arg. espécies estas, também encontradiças nas cercanias de Manaus.

A floração é anual, verificando-se esta entre junho e novembro e a frutificação, entre dezembro e março do ano seguinte.

\section{DADOS SOBRE A MADEIRA}

Macroscòpicamente, a madeira tem as mesmas características do gênero, apesar de à primeira vista parecer distinta especialmente quanto à côr e densidade. Suas características gerais são as seguintes :

\section{Aspecto geral}

Madeira muitíssimo pesada (cerne : 1,1-1,3 $\mathrm{g} / \mathrm{cm}^{3}$ sêco ao ar), muito dura e compacta; alburno amarelado de $3-4 \mathrm{~cm}$ de espessura, contrastado do cerne pardo-escuro, uniforme; textura fina; grã direita; cheiro e gôsto indistintos; superfície lisa ao tato e lustrosa; não muito difícil de trabalhar; recebe um acabamento esmerado e ótimo polimento; provàvelmente, de durabilidade elevada, resistindo bem ao ataque de insetos e à decomposição.

\section{Aspecto macroscópico (fig. 4)}

Parênquima pouco contrastado, indistinto a ôlho nu mas visivel sob lente em linhas muito finas, aproximadas e sinuosas (7-10 por $\mathrm{mm}$ ) ordenadas e contínuas, formando com os raios um reticulado uniformemente distribuído, às vêzes interrompidas pelos poros; poros pouco distintos a ôlho a nu, poucos a muito pouco numerosos, $\left(0-4\right.$ por $\left.\mathrm{mm}^{2}\right)$, pequenos $(0,1-0,2 \mathrm{~mm}$ de diâmetro), alguns médios até $0,3 \mathrm{~mm}$, solitários 


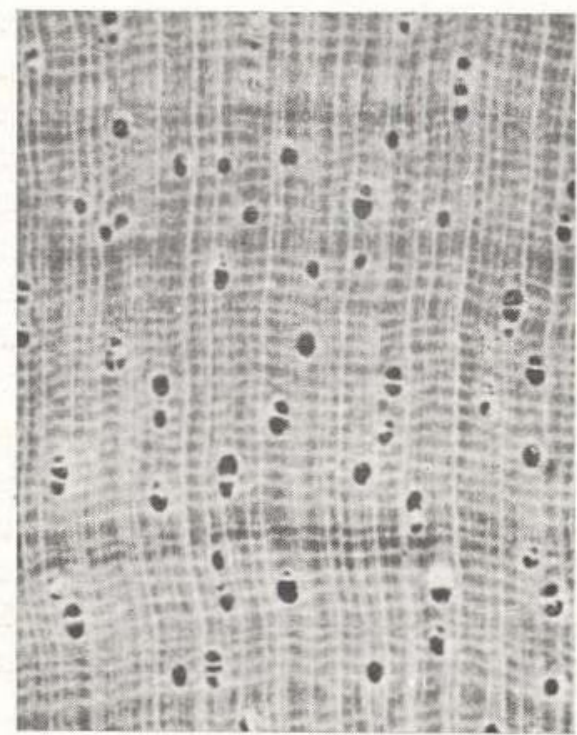

Fig. 4 - Aspecto macroscópico da madeira de Micrandra scleroxylon W. Rodr. $\mathrm{n}$. sp., em seção transversal com $10 \mathrm{X}$ de aumento. (W. Rodrigues \& A. Loureiro, 7065)

predominantes e múltiplos até 5 , vazio a maioria no alburno e geralmente obstruidos no cerne; raios muito finos, numerosos (3-7 por $\mathrm{mm}$ ) com certa uniformidade no espaçamento e espessura, distintos apenas sob lente no tôpo, na face tangencial irregularmente dispostos, pouco distintos sob lente, na face radial contrastados por linhas mais escuras; linhas vasculares finas e longas; camadas de crescimento demarcadas por zonas e fibrosas mais escuras.

\section{Aplicações}

É de valor comercial atual um pouco restrito devido não ser a espécie ainda bem conhecida regionalmente, apesar de sua relativa abundância nas matas das cercanias de Manaus. Ocasionalmente, tem sido usada para estacas, esteios, moirões, suumba de sararaca, recomendando-se também o seu emprêgo para tacos para soalho, parquetes, construção civil e naval dormentes, etc.

Localmente é conhecida por "acapuri", nome êste derivado de "acapu" (Vouacapoua americana Aub!., Leguminosae), madeira de grande valor comercial do Pará, à qual se parece.

\section{SUMMARY}

The author describes in this paper a new species of the family Euphorbiaceae (Micranda scleroxylon W. Rodr. n. sp.), a relatively abundant tree of the upland forest near Manaus, in the brazilian State of Amazonas. Some ecological data are presented for this new species, including also the gross and macroscopic features of its wood.

\section{BIBLIOGRAFIA CITADA}

PUNT, W

1962 - Pollen morphology of the Euphorbiaceae with special reference to taxonomy. Meded. Bot. Mus. \& Herb., 185: 1-116.

RECORD, J. S. \& HESS, R. W.

1949 - Timbers of the new world. New Haven, Yale Univ. Press. $640 \mathrm{p}$

RODRIgues, W. A .

1967 - Inventário florestal pilôto ao longo da estrada Manaus-Itacoatiára, Estado do Amazonas : dados preliminares. Atas Simp. sôbre Biota Amaz., 7: (Conservaşão na natureza e recur. sos naturais) : $257-267$

jCHULTES, R. E

1952 - Studies in the genus Micrandra. I - The relationship of the genus Cunuria to Micrandra. Bot. Mus. Leafl. Harv. Univ. (reprints) 15(8): : 201-221.

1955 - A new generic concept in the Euphorbiaceae. Bot. Mus. Leafl. Harv. Univ, 17(1).: 27-36. 